\title{
Spatio-temporal imaging of voltage pulses with an ultrafast scanning tunneling microscope
}

Jensen, Jacob Riis; Keil, Ulrich Dieter Felix; Hvam, Jørn Märcher

Published in:

Applied Physics Letters

Link to article, DOI:

$10.1063 / 1.118976$

Publication date:

1997

Document Version

Publisher's PDF, also known as Version of record

Link back to DTU Orbit

Citation (APA):

Jensen, J. R., Keil, U. D. F., \& Hvam, J. M. (1997). Spatio-temporal imaging of voltage pulses with an ultrafast scanning tunneling microscope. Applied Physics Letters, 70(20), 2762-2764. https://doi.org/10.1063/1.118976

\section{General rights}

Copyright and moral rights for the publications made accessible in the public portal are retained by the authors and/or other copyright owners and it is a condition of accessing publications that users recognise and abide by the legal requirements associated with these rights.

- Users may download and print one copy of any publication from the public portal for the purpose of private study or research.

- You may not further distribute the material or use it for any profit-making activity or commercial gain

- You may freely distribute the URL identifying the publication in the public portal 


\title{
Spatio-temporal imaging of voltage pulses with an ultrafast scanning tunneling microscope
}

\author{
Jacob R. Jensen, Ulrich D. Keil,, and Jørn M. Hvam \\ Mikroelektronik Centret, DTU Building 345 East, DK-2800 Lyngby, Denmark
}

(Received 13 January 1997; accepted for publication 25 March 1997)

\begin{abstract}
Measurements on an ultrafast scanning tunneling microscope with simultaneous spatial and temporal resolution are presented. We show images of picosecond pulses propagating on a coplanar waveguide and resolve their mode structures. The influence of transmission line discontinuities on the mode structure is investigated. It is also demonstrated how common and differential modes of electrical pulses are generated. The capacitive coupling between the tip and the transmission line is explained in terms of two contributions: a long range and a local coupling. We also show how these contributions affect the imaging of the propagating pulses. (C) 1997 American Institute of Physics. [S0003-6951(97)03520-1]
\end{abstract}

In 1993 Weiss et al. presented the first measurements with an optically gated tunneling microscope used to detect picosecond electrical pulses. ${ }^{1,2}$ Since then, the research in this field has concentrated on achieving a better time resolution and gaining a clearer understanding of the interaction between the tip and the sample. Subpicosecond time resolution has been achieved by Botkin et al., ${ }^{3}$ while Groeneveld et al. showed that the tip-sample interaction is due to capacitive coupling through a geometrical capacitance. ${ }^{4}$ Recently, it was shown that the linear dependence of the signal amplitude on the tunneling conductance disappears if the tip is virtually grounded instead of floating. ${ }^{5,6}$ One of the most interesting aspects of the photoconductively gated tunneling microscope is the assumed ability to combine the high spatial resolution of the scanning tunneling microscope with the high temporal resolution of optical gating techniques using femtosecond pulses. However, there has been no report on measurements with simultaneous spatial and temporal resolution until now.

In this letter, we present spatio-temporal measurements of picosecond voltage pulses on a coplanar waveguide (CPW), obtained by an ultrafast scanning tunneling microscope (USTM). The details of the setup are described in Refs. 5 and 7. The tip of the USTM is attached to one strip of a coplanar stripline and by optical excitation of a photoconductive switch between the strips, the transient tunneling current is sampled. In the present measurements, the internal preamplifier of the STM is directly connected to the electrode with the tip while an external preamplifier is connected to the other electrode, enabling measurements at a reference frequency above the cutoff frequency of the internal preamplifier. A mode-locked Ti:sapphire laser supplies 100-fs pulses at a wavelength of $800 \mathrm{~nm}$. The laser beam is split into pump and probe beams that are chopped by acoustooptic modulators at typical frequencies of $650 \mathrm{kHz}$. The pump beam generates the electrical pulses on the CPW while the probe beam performs the sampling of the transients on the tip strip lines. The delay-dependent signal is measured through the external preamplifier with a lock-in amplifier set to the difference frequency of about $20 \mathrm{kHz}$. The tip is a $5-\mu \mathrm{m} \mathrm{Pt} / \mathrm{Ir}$ wire, $\sim 200 \mu \mathrm{m}$ long, glued onto the electrode with conducting silver epoxy.

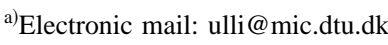

This setup allows us to move the tip in steps within the $50 \times 50 \mu \mathrm{m}$ scan area set by the range of the piezoscanner and record a delay-dependent signal for each step. In this way, a scan in space and time, or "spatio-temporal" scan, is obtained. Since the probe switch is illuminated by a fiber fixed to the tip substrate, moving the tip does not affect the sampling properties. While scanning, the STM is operated in the tunneling mode in order to prevent the tip from crashing into the surface or sticking to the sample. Measurements with the tip in contact are also done at individual tip positions. Here, the tip approaches the surface until a crash is detected as a large increase in the tunneling current. In this position, the delay-dependent signal is measured and before moving the tip to another position, it is retracted from the surface again. The sample measured throughout this letter is a CPW on a low-temperature grown GaAs substrate with an in-line gap in the center electrode. The spacings between the electrodes and their widths are $5 \mu \mathrm{m}$. The substrate was grown at $250{ }^{\circ} \mathrm{C}$, has been annealed at $600{ }^{\circ} \mathrm{C}$, and shows a carrier lifetime shorter than $400 \mathrm{fs}$, measured with differential transmission spectroscopy. With electro-optic sampling, the widths of the generated electrical pulses have been measured to be $\sim 800 \mathrm{fs}^{5}$

Figure 1 shows a grey scale contour plot of a spatiotemporal scan of an electrical pulse generated by optically exciting the in-line gap. The tip is placed $\sim 500 \mu \mathrm{m}$ away from the gap and scanned (in 50 steps) along a line perpendicular to the waveguide. A bias voltage of $50 \mathrm{mV}$ is applied to all the electrodes of the waveguide and the STM controller is set to a specific tunneling current, typically $0.3 \mathrm{nA}$. However, it is only possible to draw a tunneling current from the electrodes because the semiconducting substrate is too insulating; so for the scans between the electrodes and outside the CPW structure, the measured current is virtually zero. By measuring the tunneling current for each scan, we are thus able to map out the regions where the tip is tunneling from the electrodes, labeled "top," "center," and "bottom" in Fig. 1. The distance between the center of neighboring electrodes is $10 \mu \mathrm{m}$ which is used to calibrate the position scale on the scan images. In Fig. 1, the top line appears to be narrower than the center and bottom lines; an artifact which we think is due to a slight tilt of the sample. Because of the tilt, the piezo has come out of range in the vertical direction before reaching the edge of the electrode (the scan direction 


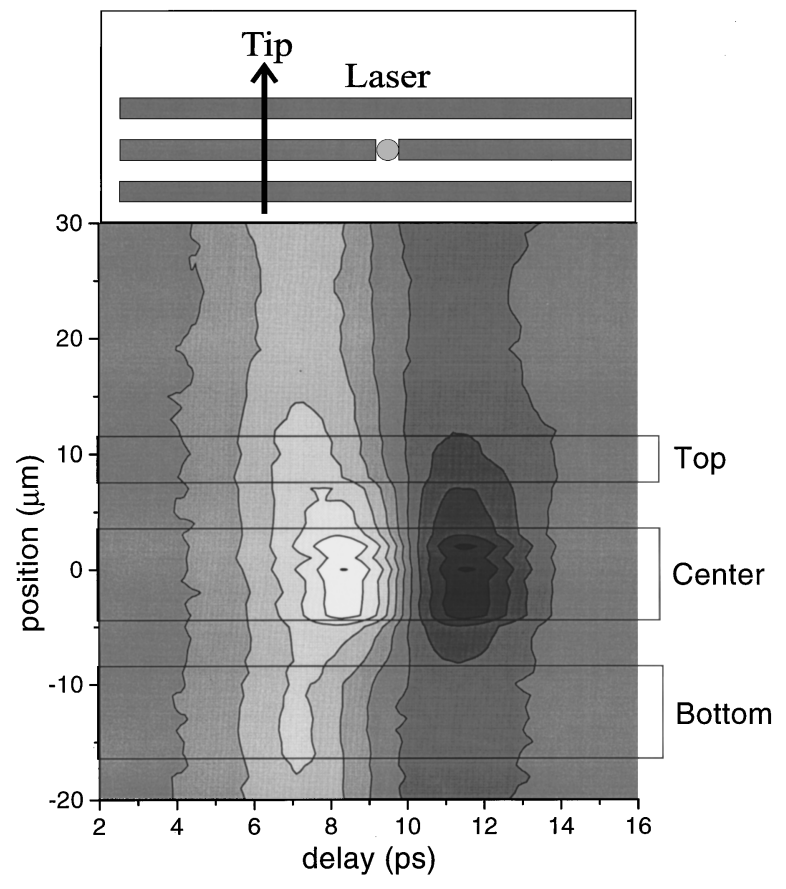

FIG. 1. Spatio-temporal scan of a voltage pulse generated by optical excitation of the in-line gap. The outline of the electrodes is indicated in the figure. The figure above the scan shows the pump beam and tip position. In the linear grey scale, white represents the areas with the maximum positive values of the signal and black the maximum negative values.

is from bottom to top). In Fig. 1, the widths of the electrodes appear broader than their spacings. We believe this effect is due to tunneling from the edge of the electrodes to the sides of the tip end that effectively increases the number of steps where the tip can draw a tunneling current from the electrodes.

For each step, two delay-dependent signals are measured, one with a switch voltage of $3 \mathrm{~V}$ across the in-line gap and one without a voltage across the gap. To suppress the signal components of nonelectrical origin (e.g., signals caused by reflected or scattered light), the signal measured without a switch voltage is subtracted from the signal measured with a voltage applied to the switch. Because the coupling from the sample to the tip is mainly capacitive, the shape of the transient signal is the derivative (with respect to delay time) of the contact measurement. ${ }^{5}$ That is, the signal measured in tunneling mode has a positive and a negative peak due to the rising and falling edge of the signal measured in contact. The pulse in the scan is a superposition of two contributions: one is confined to the center line and has a positive peak at $\tau=8.5 \mathrm{ps}$ and a negative peak at $\tau=11.5 \mathrm{ps}$ while the other extends beyond the CPW with a positive peak at $\tau=7 \mathrm{ps}$ and a negative peak at $\tau=11 \mathrm{ps}$. We refer to these two contributions as the confined and the extended signal component, respectively, and explain them with the pulse generation principle.

By illuminating an in-line gap, we generate a differential mode together with a common mode. The field lines of the differential mode run from one electrode to another, determining the voltage difference between the electrodes. The common mode originates from the change of the average voltage of the three $\mathrm{CPW}$ electrodes. The field lines of the

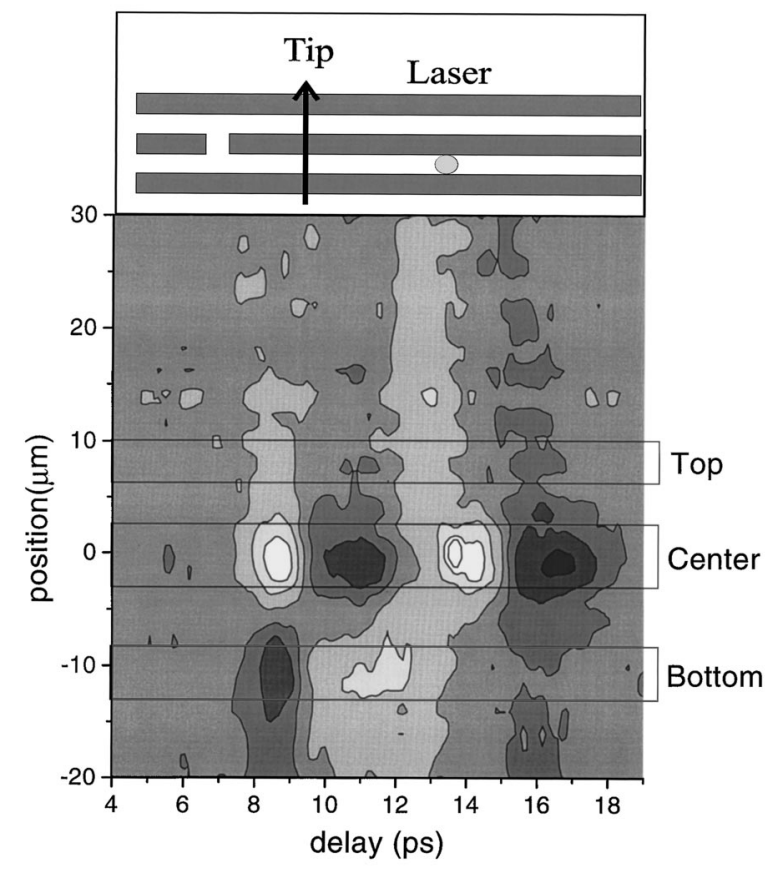

FIG. 2. Spatio-temporal scan of a voltage pulse generated by optical excitation between the center and bottom lines. The grey scale is the same as in Fig. 1 (the image has been smoothened).

common mode spread out from the electrodes to the closest external ground. There is no well-defined external ground in the setup used here, so the common mode field is expected to be spatially extended. Thus, the common mode field will propagate through the free space above the substrate to the tip with the speed of light; whereas, the speed of the guided electrical pulse is slower and is determined by the effective dielectric constant of the air and the substrate. ${ }^{8}$ The extended signal component is therefore attributed to pickup of the common mode field along the whole tip wire. The confined signal component is due to a local capacitive coupling from the transmission line to the end of the tip when the pulse passes beneath it. Because the field of the common mode spreads in all spatial directions the scan is not simply an image of the voltage pulses on the surface of the sample.

To test that the extended signal is due to a common mode, another scan was performed with the switch voltage being applied to the bottom and top lines and the pump beam exciting between the center and bottom lines (Fig. 2). Since the signal amplitude is independent of the tunneling conductance, ${ }^{7}$ scanning across differently biased lines does not affect the image. When the pump beam excites carriers in the substrate between the lines, the subsequent reduction of the voltage on the center line will balance the increase on the bottom line and only a differential mode is generated. This initial signal in the time range from $\tau=7.5 \mathrm{ps}$ to $\tau=12 \mathrm{ps}$ is indeed more confined to the lines than the signal generated with the in-line gap. In addition, there is no sign of a leading signal component. The additional delayed features in Fig. 2 show that the instrument enables the investigation of pulse propagation on transmission lines in great detail. As indicated in Fig. 2, the center line is interrupted by the in-line gap, causing the reflection seen between $\tau=12$ ps and $\tau=18$ ps. By measuring the delay-dependent signal at two different 


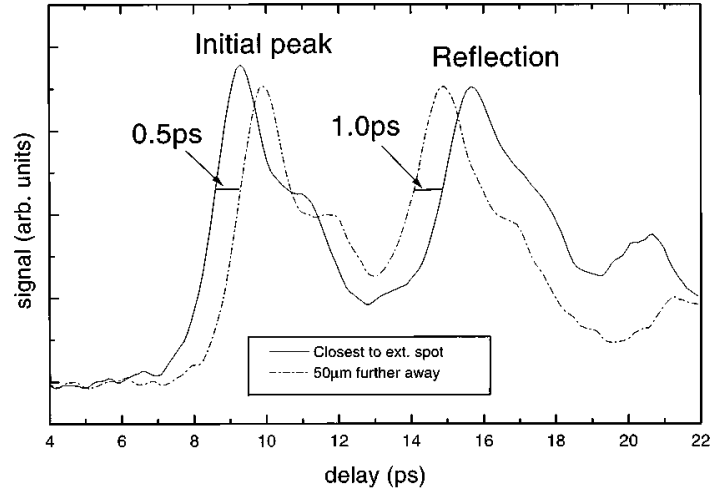

FIG. 3. Measurements of incident and reflected pulses with the tip contacting the center line at two different positions. The solid line is the measurement closest to the excitation position and the dashed line measured $50 \mu \mathrm{m}$ further away. The excitation geometry is the same as in Fig. 2.

tip positions $50 \mu \mathrm{m}$ apart on the center line (Fig. 3), we confirmed that the origin of this signal is a reflection. For these measurements the tip was in contact with the center electrode. As expected, the initial pulse arrives $\Delta \tau=0.5 \mathrm{ps}$ earlier when the tip is closest to the excitation spot and the reflection arrives $\Delta \tau=1.0 \mathrm{ps}$ later, due to an increased propagation distance of $100 \mu \mathrm{m}$. The time differences correspond very well to the propagation speed of $\sim 40 \%$ of the speed of light, calculated using an effective index method for the surrounding dielectrics of the waveguide. ${ }^{8}$ The image shows that there is no reflection on the bottom line. Therefore, the reflected pulse on the center line is not balanced by a voltage on the bottom line and has a common mode contribution. This mode is detected in the scan image as the extended pulse located at $\tau=13 \mathrm{ps}$.

Finally, we also scanned the waveguide on the other side of the gap, still generating the pulse with the pump beam between the center and bottom lines (Fig. 4). As in Fig. 1 there is an extended signal component at $\tau=8.5$ ps due to a common mode and a confined signal component on the bottom line with peaks at $\tau=9 \mathrm{ps}$ and $\tau=11.5 \mathrm{ps}$. In both experiments, the in-line gap is the source of the common mode. In the first case (Fig. 1), there is only a current pulse on the center line, due to the illumination of the in-line gap. In the second case (Fig. 4), there is only a current pulse on the bottom line because the gap has reflected the part of the incident pulse on the center line. The time difference between the confined and extended signal components is smaller in Fig. 4 than in Fig. 1 because the scan is closer to the gap. The confined signal on the bottom line in Fig. 4 is the part of the generated pulse transmitted across the gap. Measurements with the tip in contact show that there are also smaller pulses on the center and top lines. The pulses on the center and bottom lines have the same polarity whereas the pulse on the top line has opposite polarity; therefore, the signal in the scan is reduced as seen in the image. The origin of this mode structure is not well understood, but it is unlikely that it is due to unintentional pumping between the top and center lines since this would give rise to a pulse of the same polarity on the top and bottom lines.

In summary, the use of spatio-temporal scans with a USTM for resolving the mode structure of voltage pulses on

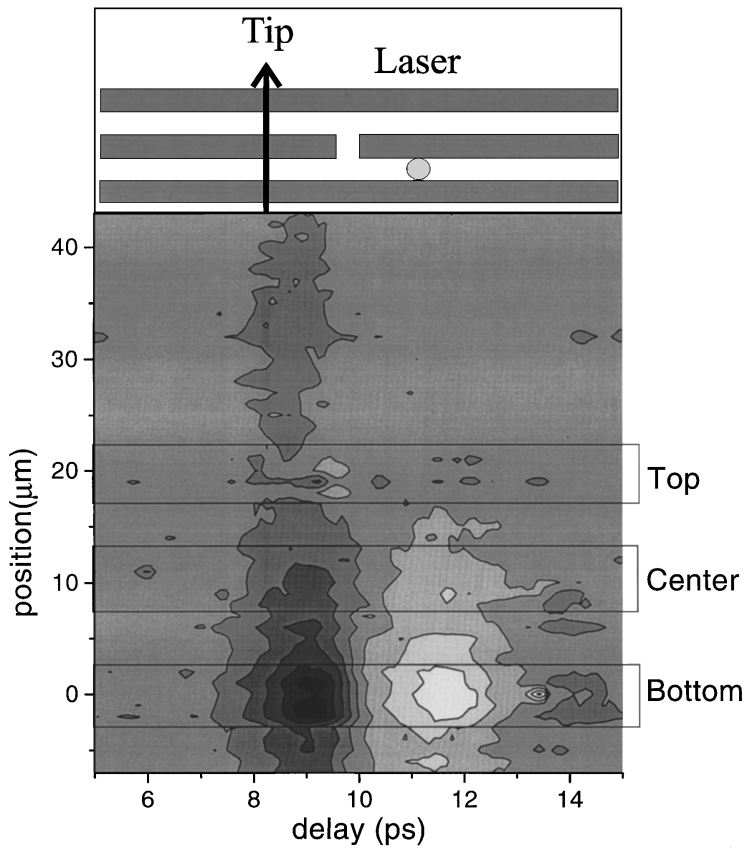

FIG. 4. Spatio-temporal scan measured on the other side of the in-line gap. The voltage pulse is generated by optical excitation between the center and bottom lines. The grey scale is the same as in Figs. 1 and 2.

a CPW is demonstrated. In particular, the reflection and transmission of a differential mode pulse incident on the inline gap is imaged. It is shown that a common mode contribution in the pulse is spread out in space whereas a purely differential mode is confined to the transmission lines in the image. None of the measurements presented here allow us to estimate the spatial resolution of the scans, but from the discussion of the capacitive coupling we conclude that the best resolution is obtained with the local coupling between the transmission line and the end of the tip. The resolution of this coupling will be of the order of the diameter of the tip and we believe that it depends on the exact shape of the tip end. As the signals are of capacitive origin, the resolution is not simply determined by the extent of the tunneling region.

The authors would like to thank Claus B. Sørensen, III-V Nanolab, for the growth of the LT-GaAs samples and Ole Hansen for fruitful discussions. This work is supported by the Danish Research Academy through a DANVIS grant and by the Danish Ministries of Research and Industry in the framework of CNAST.

${ }^{1}$ S. Weiss, D. F. Ogletree, D. Botkin, M. Salmeron, and D. S. Chemla, Appl. Phys. Lett. 63, 2567 (1993).

${ }^{2}$ S. Weiss, D. Botkin, D. F. Ogletree, M. Salmeron, and D. S. Chemla, Phys. Status Solidi B 188, 343 (1995).

${ }^{3}$ D. Botkin, J. Glass, D. S. Chemla, D. F. Ogletree, M. Salmeron, and S. Weiss, Appl. Phys. Lett. 69, 1321 (1996).

${ }^{4}$ R. H. M. Groeneveld and H. van Kempen, Appl. Phys. Lett. 69, 2294 (1996).

${ }^{5}$ U. D. Keil, J. R. Jensen, and J. M. Hvam, J. Appl. Phys. 81, 2929 (1997).

${ }^{6}$ U. D. Keil, J. R. Jensen, and J. M. Hvam, Appl. Phys. Lett. 70, 2625 (1997).

${ }^{7}$ U. D. Keil and J. M. Hvam, Technical Digest, EQEC '96, Hamburg, Germany.

${ }^{8}$ S. Ramo, J. R. Whinnery, and T. Van Duzer, Fields and Waves in Communication Electronics, 3rd ed. (Wiley, New York, 1993). 